\title{
Symbiotic 8: Parallel and Targeted Test Generation (Competition Contribution)
}

\author{
Marek Chalupa@ ${ }^{\bowtie}$, Jakub Novák, and \\ Jan Strejček(10)
}

Masaryk University, Brno, Czech Republic

$\bowtie$ Jury member and the corresponding author: chalupa@fi.muni.cz.

\begin{abstract}
The setup of Symвiотіс 8 for Test-Comp 2021 brings radical changes in the test generation for coverage-branches property. Similarly as in Symbiotic 7, we generate tests by running our fork of symbolic executor KLEE on the analyzed program. Symbiotic 8, however, runs several instances of KLEE in parallel. We run one instance of KLEE on the original program and, simultaneously, we create one (intentionally unsound) program slice for every program-terminating instruction in the program and run KLEE on these slices. Apart from this principal change, we also improved other components of the tool, mainly the program slicer. Further, our fork of KLEE now supports symbolic pointer arithmetics and comparison of symbolic addresses.
\end{abstract}

\section{Test-Generation Approach}

Symbiotic $[3,2]$ is an open-source program analysis framework that combines static analyses with code transformations in order to enable faster analysis of the code. In the setup for Test-Comp 2021, Symbiotic uses program slicing [6] in combination with symbolic execution [5].

Static (backward) program slicing [6] is a technique that removes program instructions that have no influence on reachability or the effect of selected parts of the program. In Test-Comp, we use program slicing for all properties. For coverage-error-call property, we slice the program to remove instructions that cannot affect reachability of the error location. For coverage-branches property, we use program slicing to create modified versions of the program on which we are likely to quickly generate tests that reach hard-to-cover parts of the program.

Symbolic execution [5] is a program analysis technique that enumerates all possible execution paths of a program. For every path, it computes its corresponding path condition, which is a collection of constraints on program inputs that forms the necessary and sufficient condition to follow the path. Each path condition is then used to create a test that makes the program execute the given path.

(C) The Author(s) 2021

E. Guerra and M. Stoelinga (Eds.): FASE 2021, LNCS 12649, pp. 368-372, 2021.

https://doi.org/10.1007/978-3-030-71500-7_20 


\subsection{Workflow of Symbiotic 8}

The workflow of Symbiotic 8 in Test-Comp 2021 for the property coverage-error-call is the same as in Symbiotic 7: we slice the analyzed program with respect to calls of the error function and run KLEE on this sliced program. If KLEE finds a feasible path that calls the error function, we attempt to replay this path in the unsliced program to fill in the possibly missing values returned from calls to functions __VERIFIER_nondet_* that may have been sliced away.

The workflow for the property coverage-branches changed significantly in Symbiotic 8. For this property, we run several instances of KLEE in parallel: one instance on the original program and other instances on slices generated for every terminating location in the program.

More precisely, we create a pool of processes that keeps running at most 8 processes at the same time (on the first-come-first-served basis). We start an instance of KLEE on the original program and add it to the pool. Then we identify instructions in the program that terminate the execution (further referred to as targets). For each target, we create a slice and queue a run of KLEE on this slice.

These slices are unsound in the sense that they do not preserve all execution paths to the targets. A slice is constructed in two steps:

1. We gather all instructions that are backward-reachable from the target in the target's function and recursively in the callers of the target's function. However, we move only up the call stack and do not submerge into procedures during this process.

2. After we gather all such instructions, we replace all other instructions with a call to abort and apply standard program slicing with respect to the target.

For example, consider the code on the left in Figure 1. It contains three possible targets, namely error() (line 7), abort() (line 13), and return 0 (line 17). If we slice with respect to the target error(), we start searching the program backwards from this target and get all instructions in the body of function foo. Then we pop up from the call to line 16 and collect all instructions of function main except the call to abort (from which the call to foo is unreachable). All instructions except the gathered ones are replaced with a call to abort. Standard program slicing then produces the program depicted in the middle in Figure 1 (in this case, it just removes the return). The slice for the target abort () preserves only three first lines of main as depicted on the right in Figure 1.

Whenever the main instance of KLEE finishes tests generation, we have tests for all feasible execution paths of the program. Therefore, we kill all other running instances of KLEE and discard tests that were not generated by the main instance to reduce the size of the test suite. If the main instance does not finish before timeout, we keep all generated tests.

Using the unsound slices aims only to help reaching hard-to-cover places in the program. In particular, potentially expensive detours are replaced by abort and symbolic execution thus does not waste resources to discover them (see line 2 in the middle in Figure 1). The current construction of unsound slices 

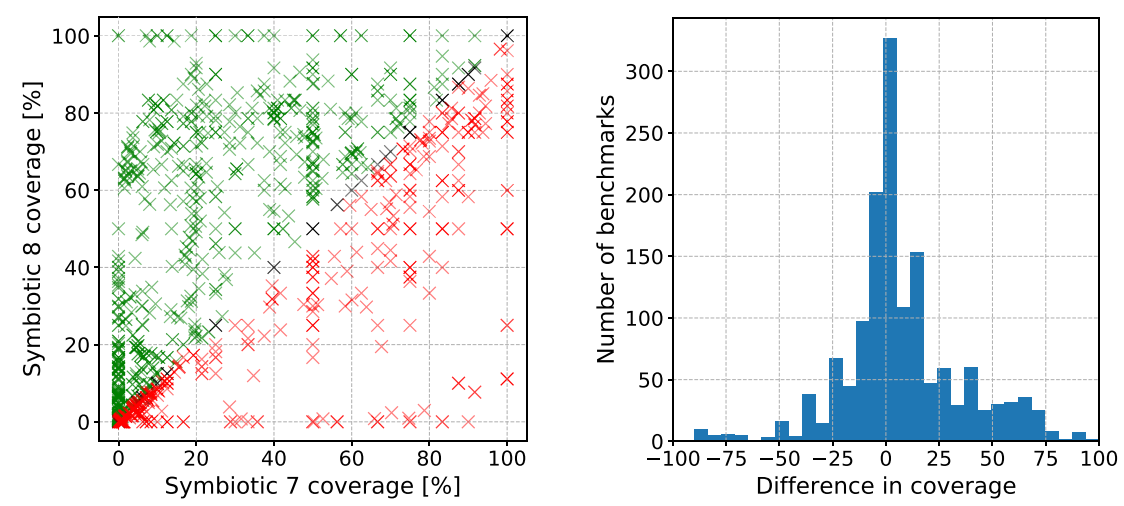

Fig. 2. The coverage achieved by Symbiotic 8 and 7 on individual benchmarks of the Cover-Branches category

program. Using unsound program slices for terminating instructions attempts to alleviate this problem. Although the slice is not guaranteed to preserve paths to the target for which it was created, there are programs where this technique helps symbolic execution to cover substantially more instructions. However, there are also many cases where the technique worsens the coverage alike.

Figure 2 illustrates the overall positive and negative effect of this approach. The scatter plot on the left compares the coverage achieved by SYMBIOTIC 8 and the coverage achieved by Symвiотіс 7 on individual benchmarks that were used in both Test-Comp 2020 and 2021. ${ }^{1}$ The scatter plot shows that the behavior of the tool changes dramatically. To summarize the data, we compute the difference between the two coverages on each benchmark (for example, if Symbiotic 8 achieves $80 \%$ and Symbiotic $760 \%$ coverage, the difference is $+20 \%$ ). The histogram on the right indicates that the overall effect of unsound slices is positive as the distribution is skewed to positive values. Indeed, SYMBIOTIC 8 won the 3rd place in the category Cover-Branches (corresponding to coverage-branches property) in Test-Comp 2021 which is a big improvement over the previous Test-Comp, where Symbiotic was 8 th out of 9 participants in this category.

The workflow of Symbiotic on coverage-error-call did not change from the last year and thus the results are similar.

\section{Tool Setup and Configuration}

The archive is available at https://doi.org/10.5281/zenodo.4491729. Run SYMBIOTIC with the following command

\footnotetext{
1 The use of unsound slices is not the only difference between Symвiотіс 8 and 7 , but we believe that it has the biggest impact on the presented results.
} 
bin/symbiotic --test-comp --prp <prpfile> [--32] <source>

where --prp sets the verified property and --32 tells SymBiotic to assume 32-bit architecture (64-bit architecture is assumed by default). The generated test-cases are stored in the directory test-suite.

\section{Software Project and Contributors}

Symbiotic 8 as it competes in Test-Comp 2021 has been developed by Marek Chalupa and Jakub Novák under the supervision of Jan Strejček. The tool and its components are available under MIT License. LLVM, KLEE, and Z3 are also available under open-source licenses. The project web page is:

$$
\text { https://github.com/staticafi/symbiotic }
$$

\section{References}

1. C. Cadar, D. Dunbar, and D. Engler. KLEE: Unassisted and automatic generation of high-coverage tests for complex systems programs. In OSDI, pages 209-224. USENIX Association, 2008. http://www.usenix.org/events/osdi08/tech/ full_papers/cadar/cadar.pdf.

2. M. Chalupa, T. Jašek, L. Tomovič, M. Hruška, V. Šoková, P. Ayaziová, J. Strejček, and T. Vojnar. Symbiotic 7: Integration of predator and more (competition contribution). In $T A C A S$, volume 12079 of $L N C S$, pages 413-417. Springer, 2020. doi: 10.1007/978-3-030-45237-7_31.

3. M. Chalupa, M. Vitovská, T. Jašek, M. Šimáček, and J. Strejček. Symbiotic 6: generating test cases by slicing and symbolic execution. International Journal on Software Tools for Technology Transfer, 2020. doi: 10.1007/s10009-020-00573-0.

4. L. de Moura and N. Bjørner. Z3: an efficient SMT solver. In TACAS, volume 4963 of LNCS, pages 337-340. Springer, 2008. doi: 10.1007/978-3-540-78800-3_24.

5. J. C. King. Symbolic execution and program testing. Communications of ACM, 19(7):385-394, 1976. doi: 10.1145/360248.360252.

6. Mark Weiser. Program slicing. IEEE Transactions on Software Engineering, 10(4):352-357, 1984. doi: 10.1109/TSE.1984.5010248.

7. LLVM. http://llvm.org/.

Open Access This chapter is licensed under the terms of the Creative Commons Attribution 4.0 International License (http://creativecommons.org/licenses/by/4.0/), which permits use, sharing, adaptation, distribution and reproduction in any medium or format, as long as you give appropriate credit to the original author(s) and the source, provide a link to the Creative Commons license and indicate if changes were made.

The images or other third party material in this chapter are included in the chapter's Creative Commons license, unless indicated otherwise in a credit line to the material. If material is not included in the chapter's Creative Commons license and your intended use is not permitted by statutory regulation or exceeds the permitted use, you will need to obtain permission directly from the copyright holder. 